\title{
Genetic Associations of Angiotensin-Converting Enzyme with Primary Intracerebral Hemorrhage: A Meta-analysis
}

\author{
Yuhao Sun ${ }^{1}$, Ye Liu' ${ }^{2}$, Lora Talley Watts ${ }^{3}$, Qingfang Sun ${ }^{1}$, Zhihong Zhong ${ }^{1}$, Guo-Yuan Yang ${ }^{1,2,4}$, \\ Liuguan Bian ${ }^{1 *}$
}

1 Department of Neurosurgery, Ruijin Hospital, Shanghai Jiao Tong University School of Medicine, Shanghai, China, 2 Department of Neurology, Ruijin Hospital, Shanghai Jiao Tong University School of Medicine, Shanghai, China, 3 Research Imaging Institute, University of Texas Health Science Center at San Antonio, San Antonio, Texas, United States of America, 4 Neuroscience and Neuroengineering Research Center, Med-X research Institute, Shanghai Jiao Tong University, Shanghai, China

\begin{abstract}
Background: A number of studies have reported an association of angiotensin-converting enzyme (ACE) gene polymorphism with primary intracerebral hemorrhage (PICH), however the reports have demonstrated inconclusive results. To clarify this conflict, we updated the previously performed meta-analysis by Peck et al., which revealed negative results, by investigating the ACE polymorphism and its correlation to $\mathrm{PICH}$.

Methods: PubMed and Embase databases (through Dec 2012) were searched for English articles on the relationship of the I/ D polymorphism in ACE with PICH in humans. Summary odds ratios (ORs) were estimated and potential sources of heterogeneity and bias were explored.

Results: A total of $805 \mathrm{PICH}$ cases and 1641 control cases obtained from 8 case-control studies were included. The results suggest that in dominant genetic models, the ACE I/D polymorphic variant was associated with a $58 \%$ increase in susceptibility risk of PICH (OR $=1.58 ; 95 \% \mathrm{Cl}=1.07-2.35$ for $\mathrm{DD}$ vs. $\mathrm{DI}+\mathrm{II})$. However, in the subgroup analysis based on race, a significant increased risk was found in Asian DD homozygote carriers (OR $=1.76$ and $95 \% \mathrm{Cl}=1.16-2.66$ for DD vs. $\mathrm{DI}+\mathrm{II})$, but not in Caucasian DD homozygote carriers $(\mathrm{OR}=1.18,95 \% \mathrm{Cl}=0.36-3.88, \mathrm{P}=0.784$ for $\mathrm{DD}$ vs. DI+II). The heterogeneity between studies was remarkable, and its major sources of heterogeneity were due to the year in which the study was published. No potential publication bias was observed in dominant genetic models.
\end{abstract}

Conclusions: These data demonstrated evidence of a positive association between ACE I/D polymorphism with PICH, and suggested that the ACE gene is a PICH susceptible gene in Asian populations.

Citation: Sun Y, Liu Y, Watts LT, Sun Q, Zhong Z, et al. (2013) Genetic Associations of Angiotensin-Converting Enzyme with Primary Intracerebral Hemorrhage: A Meta-analysis. PLoS ONE 8(6): e67402. doi:10.1371/journal.pone.0067402

Editor: Stefano L. Sensi, University G. D'Annunzio, Italy

Received March 8, 2013; Accepted May 18, 2013; Published June 27, 2013

Copyright: ( 2013 Sun et al. This is an open-access article distributed under the terms of the Creative Commons Attribution License, which permits unrestricted use, distribution, and reproduction in any medium, provided the original author and source are credited.

Funding: Engineering Cross Research fund: YG2012MS06 (YHS). The funders had no role in study design, data collection and analysis, decision to publish, or preparation of the manuscript.

Competing Interests: The authors have declared that no competing interests exist.

*E-mail: bian6612@yahoo.com.cn

9 These authors contributed equally to this work.

\section{Introduction}

Primary intracerebral hemorrhage $(\mathrm{PICH})$ represents about $15 \%$ of all occurring strokes, and has much higher mortality rate than ischemic stroke. In the United States approximately 20,00040,000 new cases of PICH emerge each year [1,2], with more than $50 \%$ of these patients resulting in death or severe disability, even when provided with best medical care [3]. Epidemiological studies have highlighted differences in the incidence of PICH between different ethnicities [4], with African Americans having twice the incidence rate and Japanese having four times the incidence rate than the Caucasian population [5]. Although hypertensive arteriolosclerosis and cerebral amyloid angiopathy account for the majority of sporadic PICH, a growing spectrum of genetic risk factors have more recently been identified. Characterization of these genetic variations may allow for improved prognostication and prevention of PICH.
Growing evidence implicates angiotensin-converting enzyme (ACE), a key enzyme of the rennin-angiotensin system, as an important modulator of cerebrovascular disease (CVD). The ACE gene is located on chromosome 17q23 and consists of 26 exons and 25 introns. A crucial polymorphism termed Ins/Del occurs due to an insertion (I) or deletion (D) of 287 base pairs within intron 16. The $\mathrm{D}$ allele of the polymorphism accounts for significantly higher serum ACE levels and activity, compared to carriers of the insertion allele [6]. Additionally, increased serum levels of ACE may contribute to vascular injury, which can further lead to CVD. There have been several reports on the association of the ACE gene polymorphism with atherosclerosis [7]. Moreover, ACE has been shown to inhibit atherosclerosis progression [8]. Although recent data lack significant evidence of neuroprotection in patients with ICH [9], these findings imply that the ACE gene may be related to a predisposition to ICH. 
In view of few modifiable risk factors for PICH, acute therapeutic options are limited to controlling hypertension. Improving risk assessments through the identification of genetic variants in ICH may contribute to prevention efforts. A previous systematic review of hemorrhagic stroke, published in 2008, failed to confirm a significant and consistent association with ACE I/D [10], likely due to low statistical power and the small number of studies utilized in this study. Moreover, the evidence was controversial, and as a result scholars have become engaged to provide novel views on this issue recently.

Here, we conducted a comprehensive literature-based metaanalysis in which we updated the sample size and explored the ACE I/D variants association with PICH.

\section{Methods}

\section{Data Sources and Searches}

We independently searched papers using Medline (PubMed) and Embase through Dec 2012 to identify eligible genetic association studies evaluating ACE genotype and PICH. Search terms included hemorrhagic stroke, intracerebral hemorrhage and cerebral amyloid angiopathy, in combination with polymorphism or SNP. Search results were limited to articles of human studies written in English. All references cited in the identified publications were also searched for additional studies not indexed in these two databases. Case reports, editorials, and review articles were excluded.

\section{Study Selection and Eligibility Criteria}

The inclusion criteria were: (1) case-control studies and cohorts which evaluated the association between ACE I/D polymorphism and PICH risk; (2) studies using validated molecular methods for genotyping; and (3) studies containing independent data of ACE I/D genotype frequency for estimating an odds ratio (OR) with a 95\% confidence interval (CI). Cases were confirmed with diagnosis of intracerebral hemorrhage based on neuroimaging (magnetic resonance imaging or computerized tomography). Control populations: The vast majority of subjects classified as 'controls' were not subjected to neuroimaging, based on the lack of symptoms present following a clinical assessment for hemorrhage.

Studies were excluded if (1) the patients were children (age $<18$ years), (2) the genotype frequency was not adequately reported (and such data could not be obtained from the authors), (3) a case was caused by trauma, hemorrhagic rupture of a tumor or arteriovenous malformation, (4) the study focused solely on intracranial hemorrhage following acute ischemic stroke.

For studies containing overlapping cases or controls, the most recent and/or largest study with extractable data was included to avoid double counting.

\section{Data Extraction}

From each selected study the following information was extracted: name of first author, journal, year of publication, country, ethnicity, demographic characteristics, number of ICH cases and controls, confirmation of diagnosis, genotyping method, genotype distributions and allele frequency, and whether genotypes were stated to be in Hardy-Weinberg equilibrium (HWE). If the frequencies of the alleles and/or the genotypic distributions were unavailable, detail information was calculated from the papers. We did not contact individual authors for further information.

The initial data extraction was undertaken by Y Liu. Several subsequent passes were then confirmed by Y Sun to ensure the comprehensive inclusion of all appropriate studies. All studies were adjudicated by 2 independent reviewers ( $Q$ Sun and Z Zhong), with disagreements resolved through discussion and consensus.

\section{Assessment of Study Quality}

Two investigators (Y Liu and Y Sun) independently rated the quality of each retrieved study using a 10-point scoring system developed for this meta-analysis. The scoring system was based on factors that we believed would be indicators of high quality observational studies (Table 1). Study design, study size, source of population, genotyping method, genotypes stated to be in HWE and adjusted ORs were included in our evaluation of the quality of each assessed study. We assessed each study against a checklist of key quality indicators, however, studies were not included or excluded on the basis of these quality indicators.

\section{Statistical Analysis}

A list of details extracted from each study is included in Table 2. We performed a meta-analysis to investigate the association between ACE I/D and PICH for the allele contrast (D vs I), and the recessive (DD vs ID and II) and dominant (DD and ID vs II) models as previously utilized [11]. To measure the strength of association between ACE I/D polymorphism and ICH risk, overall odds ratios (OR), with the corresponding 95\% confidence intervals (CI) were assessed. OR was calculated by a fixed-effects model using the Mantel-Haenszel method [12,13] or the randomeffects model using the DerSimonian and Laird method [14] according to the heterogeneity. Statistical heterogeneity across the various studies was determined by the Cochran's $\mathrm{X}^{2}$ based $\mathrm{Q}$ statistic and $\mathrm{I}^{2}$ with statistical significant considered at $\mathrm{P}<0.10$ [15-17]. When the $\mathrm{P}$ value was greater than 0.10 , the fixed-effects model was used to pool the data, otherwise, a random-effects model was utilized. The significance of the pooled OR were determined by $\mathrm{Z}$-test, with a $\mathrm{P}$ value less than 0.05 considered statistically significant. When we searched the HapMap database, we identified different linkage disequilibrium groups around the ACE gene among different ethnicities. Therefore, Caucasian and Asian participants were separated according to additional sensitivity analysis.

The Begg's funnel plots and Egger's test were conducted to statistically assess potential publication bias (using a $\mathrm{p}$ value of $<0.05$ ) [18]. All statistical tests were performed using STATA 10.0 software (StataCorp, College Station, TX, USA). We prepared this report in accordance to the Meta-analysis of Observational Studies in Epidemiology reporting guidelines [19].

\section{Results}

\section{Search Results}

A flow diagram of the performed database search is shown in Figure 1. Combining PubMed and Embase searches yielded 123 studies that were further screened using titles, abstracts, and keywords. According to the inclusion and exclusion criteria, we included 8 studies for meta-analysis of the association between ACE I/D polymorphism and PICH [20-27]. The complete data set included 1641 healthy people from the general population and 805 patients with primary intracerebral hemorrhages.

\section{Study Characteristics}

The included studies were published from 1996 through 2011. All 8 studies evaluated susceptibility to PICH. The characteristics of each study are summarized in Table 3 . The majority of studies were conducted on Asian populations and the remaining 2 studies were conducted on Caucasian populations. Studies had a mean of 480.6 (range 192 to 698 ) total participants. The mean age of 


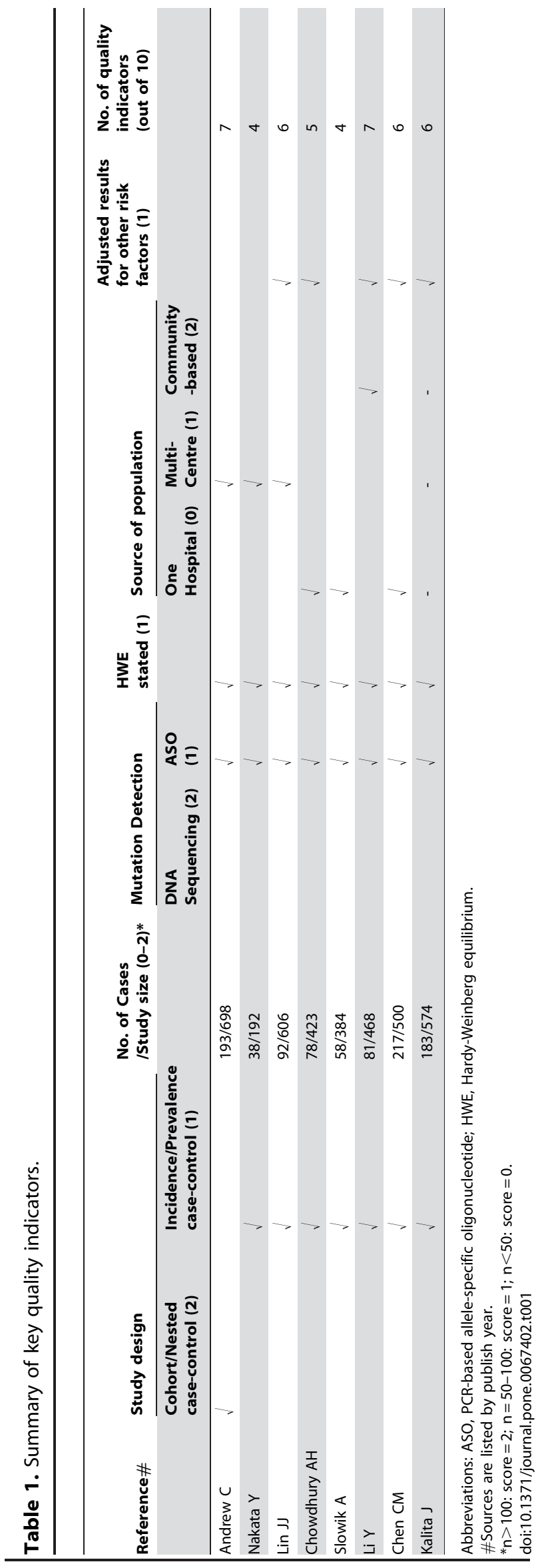

patients diagnosed with PICH ranged from 55 to 74 years. 5 out of the 8 studies used frequency-matched controls based on gender and/or age. The larger studies generally fulfilled more of our quality indicators for PICH genetics than the smaller studies (table 1). The genotype distributions among the controls were in agreement with HWE for all included studies.

\section{Quantitative Data Synthesis}

Genotype and allele distributions for each study are shown in Table 2. In Figure 2, the heterogeneity of dominant, recessive model and allele model for all eight studies was analyzed. In Figure 2, the I-square value was $62.7 \%$ for the dominant model, $68.1 \%$ for the recessive model and $75.4 \%$ for allele contrast, respectively, suggesting moderate $(50-75 \%)$ and high $(>75 \%)$ heterogeneity. The main analysis for investigating the association between the DD genotype and the risk of PICH relative to the DI/ II genotype revealed significant heterogeneity $(\mathrm{P}=0.09)$ among the 8 studies using the dominant model. Additionally, the pooled random effects $\mathrm{OR}$ was significant $(\mathrm{OR}=1.582$ [95\% CI, 1.0672.347]). However, the recessive and allele models showed no significant association (random effects ORs, 1.254 [95\% CI, 0.871-1.806] and 1.273 [95\% CI, 0.972-1.667], respectively, Figures S1 \& S2). The additive and codominant model produced a non-significant association ( $\mathrm{OR}=1.645$ [95\% CI, 0.961-2.816], $\mathrm{OR}=0.900 \quad[95 \% \mathrm{CI}, 0.678-1.197]$ ) as anticipated (figure not shown).

In addition, the $\mathrm{Z}$ value for the test for overall effect was 2.28 $(\mathrm{P}=0.022)$ for $\mathrm{DD}$ vs. DI+II model, $1.22(\mathrm{P}=0.224)$ for $\mathrm{DD}+\mathrm{DI}$ vs. II model, $1.75(\mathrm{P}=0.080)$ for $\mathrm{D}$ allele vs. I allele, suggesting that subjects who carry the DD genotype are more vulnerable to PICH compared to those in the dominant model. Overall, the results suggest that the variant genotypes of ACE I/D polymorphism were associated with a significantly higher risk of PICH when all the eligible studies were pooled into the meta-analysis.

\section{Subgroup Analyses}

To control influence by ethnic heterogeneity, we separated the studies into those with either Caucasian or Asian samples. In the two studies using Caucasian subjects, the patient population was comprised of $106 \mathrm{PICH}$ patients and 331 healthy controls, and we found significant heterogeneity among the $\mathrm{ORs}\left(\mathrm{P}_{\text {dominant }}=0.018\right.$, $\left.P_{\text {recessive }}=0.070, P_{\text {allele }}=0.006\right)$. The pooled OR from the two studies did not show a significant association for any genetic model $\left(\mathrm{P}_{\text {dominant }}=0.784, \mathrm{P}_{\text {recessive }}=0.780, \mathrm{P}_{\text {allele }}=0.945\right.$, Figures $2, \mathrm{~S} 1 \&$ S2). In the 6 Asian studies, comprised of 699 PICH patients and 1310 healthy controls, there was remarkable heterogeneity among the ORs. Asian subjects showed obvious increased risk under a dominant, recessive and allele model with a magnitude of effects similar to that of the main analysis. $\left(\mathrm{P}_{\text {dominant }}=0.008, \mathrm{P}_{\text {reces- }}\right.$ sive $=0.070, \mathrm{P}_{\text {allele }}=0.020$, Figures $\left.2, \mathrm{~S} 1 \& \mathrm{~S} 2\right)$.

\section{Study Quality}

The meta regression in the dominant model demonstrated that the major source of heterogeneity was the publication year $(\mathrm{P}=0.032)$. Although ethnic background produced a significant association, the ethnic effect in the meta regression was not significant $(\mathrm{P}=0.483)$. For the gender difference, we found more males than females were included in the meta-analysis for both PICH patients and controls. There was no significant effect of gender in the meta regression analysis $(\mathrm{P}=0.958)$. 
Table 2. The distribution of the ACE I/D variant for cases and controls.

\begin{tabular}{|c|c|c|c|c|c|c|c|c|c|c|}
\hline \multirow[t]{3}{*}{ Reference } & \multicolumn{6}{|c|}{ Distribution of genotype } & \multicolumn{4}{|c|}{ Distribution of allele } \\
\hline & \multicolumn{2}{|l|}{ II } & \multicolumn{2}{|l|}{ ID } & \multicolumn{2}{|l|}{ DD } & \multicolumn{2}{|l|}{ I } & \multicolumn{2}{|l|}{ D } \\
\hline & Case & Control & Case & Control & Case & Control & Case & Control & Case & Control \\
\hline Andrew C & 17 & 50 & 21 & 102 & 10 & 63 & 55 & 202 & 41 & 228 \\
\hline Nakata Y & 18 & 11 & 17 & 22 & 3 & 5 & 53 & 44 & 23 & 32 \\
\hline Lin JJ & 31 & 113 & 43 & 142 & 18 & 45 & 105 & 368 & 79 & 232 \\
\hline Chowdhury AH & 32 & 79 & 37 & 97 & 9 & 14 & 101 & 255 & 55 & 125 \\
\hline Slowik A & 10 & 27 & 22 & 57 & 26 & 32 & 42 & 111 & 74 & 121 \\
\hline Chen CM & 85 & 159 & 108 & 101 & 24 & 23 & 278 & 419 & 156 & 147 \\
\hline Li Y & 8 & 59 & 32 & 139 & 41 & 113 & 48 & 257 & 114 & 365 \\
\hline Kalita J & 39 & 64 & 89 & 102 & 65 & 22 & 167 & 230 & 219 & 146 \\
\hline
\end{tabular}

\section{Sensitivity Analysis}

To assess the stability of the results of the current meta-analysis, we performed sensitivity analysis by sequentially excluding each study. The pooled ORs ranged from 1.40 to 1.82 in the dominant model, and statistically similar results were obtained after sequentially excluding each study except for the Slowik's or Li's study. The pooled ORs ranged from 1.14-1.42 in the recessive model, and statistically similar results were obtained after sequentially excluding each study except for the Catto study. The pooled ORs ranged from 1.18-1.41 in the allele contrast model, and statistically similar results were obtained after sequentially excluding each study except for the Catto or Nakata studies. These results suggested that the stability of our metaanalysis is affected by these four studies.

\section{Publication Bias}

Publication bias was assessed by the Begg's funnel plot and Egger's test. Visual inspection of the shape of the funnel plots showed symmetry in the DD vs. II+DI comparison genetic model, suggesting the absence of publication bias (Figure 3). Subsequently, the Egger's test was performed to provide statistical evidence of the funnel plot asymmetry. The results indicated the current metaanalysis demonstrated a lack of publication bias using the dominant and recessive models, but did exist using the allele model $(\mathrm{t}=-0.160, \mathrm{P}=0.160$ for $\mathrm{DD}$ vs $\mathrm{DI} / \mathrm{II} ; \mathrm{t}=-1.78$,

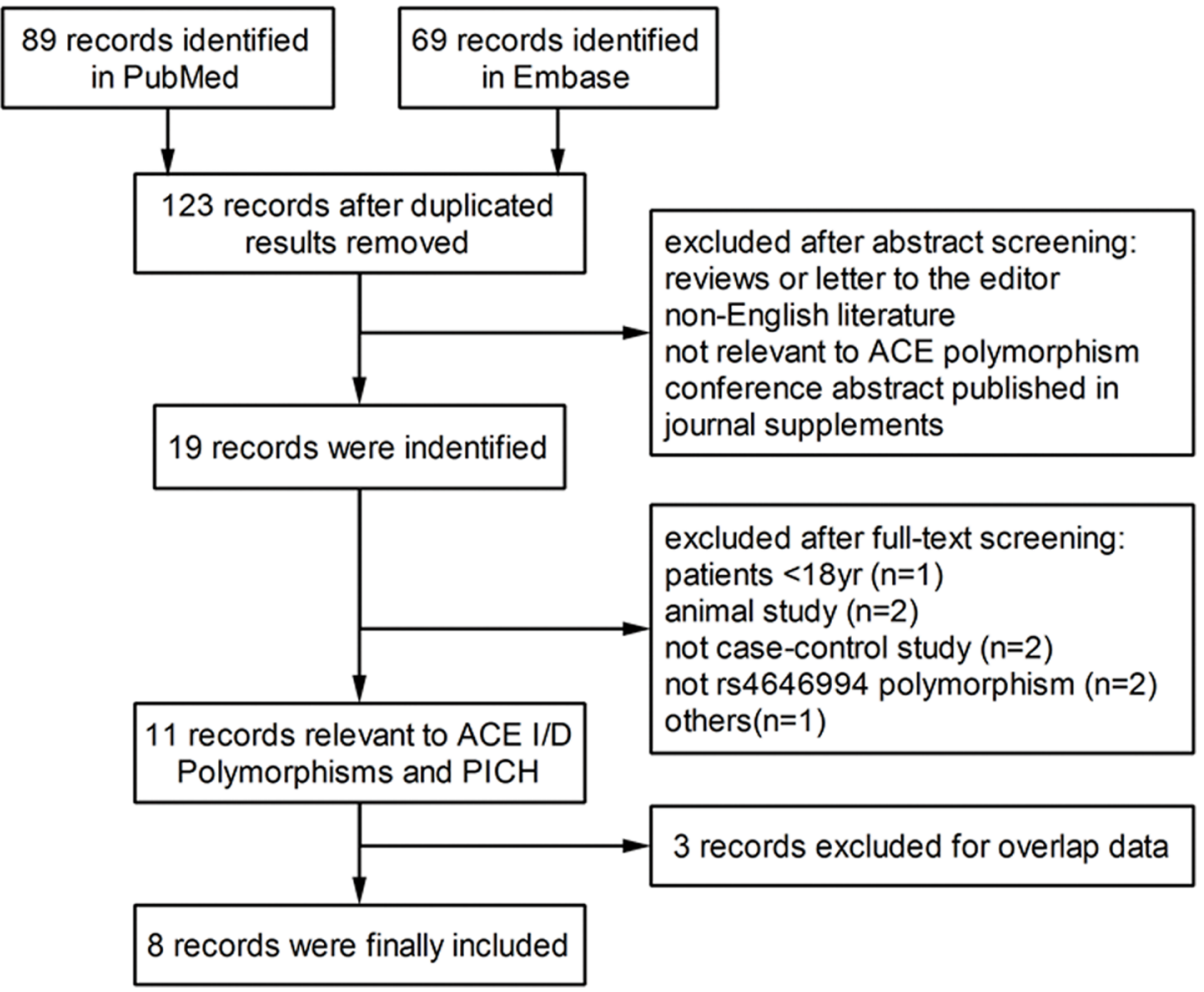

Figure 1. Study selection and exclusion. Flowchart of retrieved and excluded studies, with specification of the reasons. doi:10.1371/journal.pone.0067402.g001 


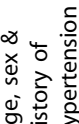

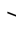

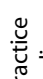
을

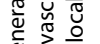

ब,

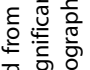

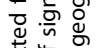

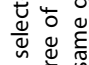

हो)

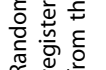

要学 $\cong$

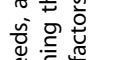

$\Phi$

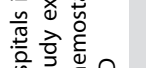

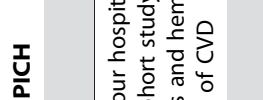

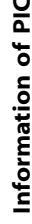

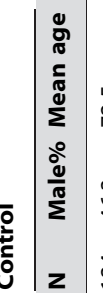

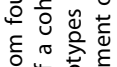

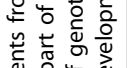

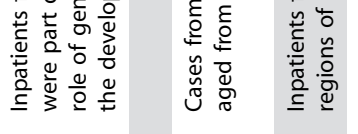

क्षे

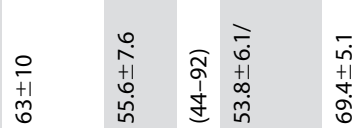

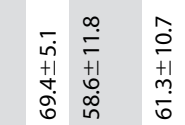

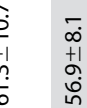

की

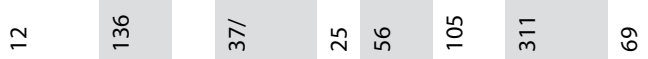

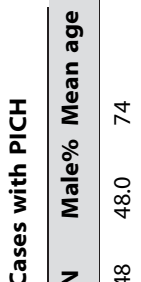

|

$\frac{0}{\frac{0}{0}}$

4

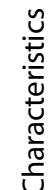

$\frac{\sqrt[U]{\frac{\pi}{0}}}{\frac{0}{0}}$

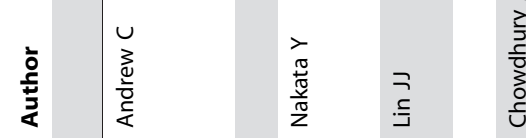

$\frac{\sqrt{0}}{\frac{0}{6}}$

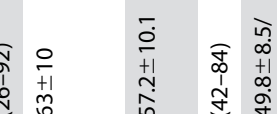

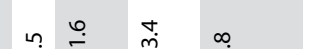

ô. i্d

$\underset{\infty}{+\infty}$

$\stackrel{\infty}{m}$

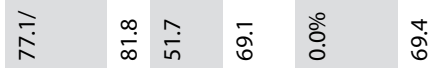

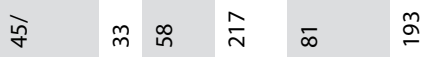

息

$\frac{\sqrt{2}}{\frac{5}{0}}$

$\frac{\pi}{4}$

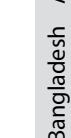

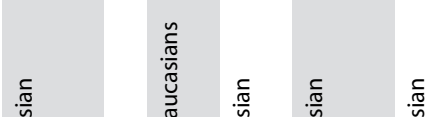

先

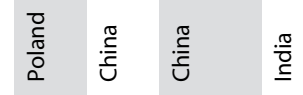

준

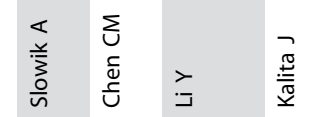

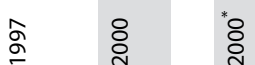

ป⿱艹 


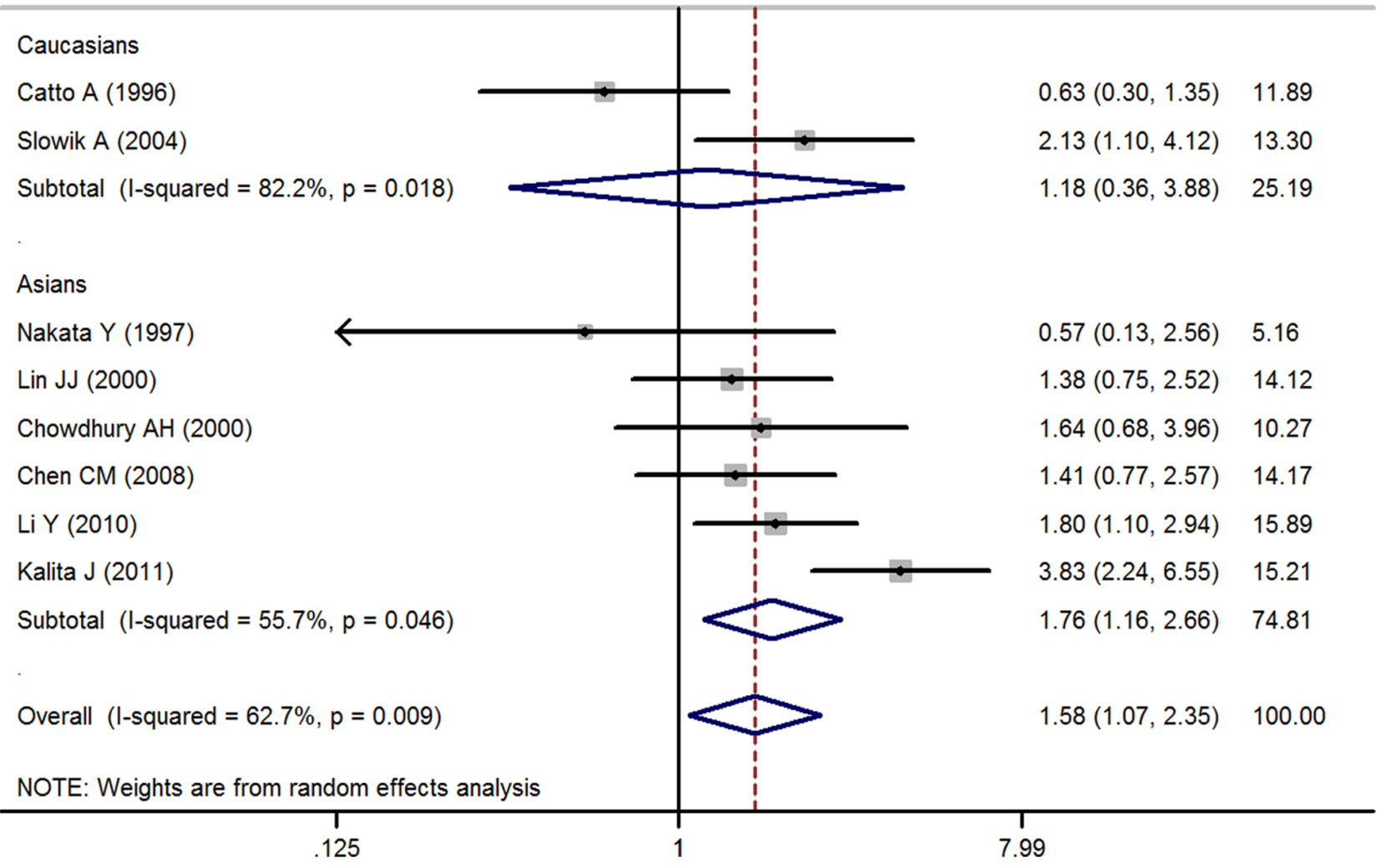

Figure 2. Meta-analysis with a random-effects model for the association between PICH risk and the ACE I/D polymorphism: subgroup analysis by race (dominant model). doi:10.1371/journal.pone.0067402.g002

$\mathrm{P}=0.125$ for $\mathrm{DD} / \mathrm{DI}$ vs II; $\mathrm{t}=-2.49 \mathrm{P}=0.047$ for $\mathrm{D}$ vs I, Figures S3 \& S4).

\section{Discussion}

The renin angiotensin system has been implicated in the pathophysiology of PICH by accelerating atherosclerosis [28]. Inactive angiotensin I can be transformed into vasoactive and idosterone-stimulating peptide angiotensin II through ACE, the key enzyme of the RAS [29]. A previous meta-analysis failed to detect an association between the ACE I/D polymorphism and PICH susceptibility [10]. The present meta-analysis explored a much larger sample size, and suggested a positive association between the ACE I/D polymorphism and PICH risk.

The use of composite quality scales may produce inherent problems and make it difficult to interpret the results, however the scale seemed to provide an overall assessment when comparing studies [30]. PICH is common in the aging population since the incidence of PICH increases gradually with age [31]. Previous reviews of sex differences in stroke epidemiology suggest that the incidence rates of intracerebral hemorrhage are higher among men [32]. Hence, if information of these risk factors were limited and could not be determined from the original studies, the association between ACE mutations and PICH risk could not to be congruently explained in the risk-unmatched, low-quality studies, compared to the age and sex-matched, high-quality studies. Given that heterogeneity comes from variations in study quality to a great extent [33-35] we should mention that significant heterogeneity existed in the overall comparison among the different genetic models. Ideally, age, sex and stable/ exacerbation situation of hypertension should be matched in all cases and controls.

Considering that the role of ACE in the pathogenesis of cerebrovascular lesions remains unclear, our meta-analysis findings may account for the relationship of ACE polymorphism with PICH. The ACE D allele has been described to be associated with $28-47 \%$ of the variance of ACE activity both in the circulation [6,36] and in tissues [37]. Increased levels of angiotensin II, converted by ACE, results in early arteriolar proliferation of smooth muscle [38],followed by smooth muscle cell death and collagen deposition [39]. This process impairs vasoconstriction and results in hypertension, which worsens arteriosclerosis, eventually leading to possible intracerebral hemorrhage. Moreover, chronic exposure to high levels of plasma ACE may contribute to increased vascular wall thickness and stiffness, which increases cerebrovascular risk as well [40]. These factors indicate that the genotype of the ACE I/D polymorphism are associated with higher PICH risk.

Subgroup analysis revealed a significant increased risk of PICH among Asians, but not in Caucasians, suggesting that ethnic differences in genetic backgrounds and the environment they lived in may be involve in the incidence of PICH. There are a number of factors that might explain the differences seen in the two populations studied. First, genetic backgrounds (SNP frequency) 


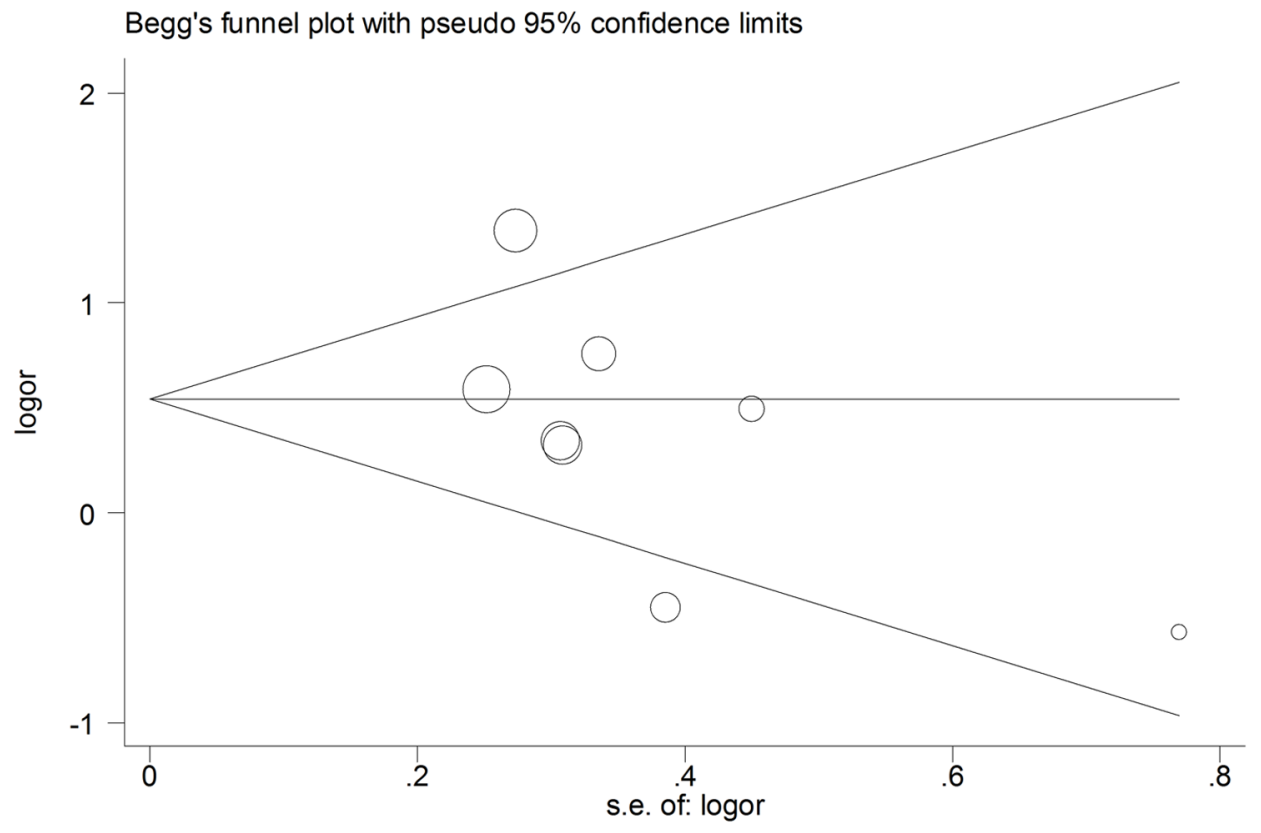

Figure 3. Begg's funnel plot for publication bias in selection of studies of the ACE I/D polymorphism (dominant model). doi:10.1371/journal.pone.0067402.g003

vary greatly from Asians to Caucasians. Second, the difference in living environments between these two populations may account for the different genetic effects. Thus, additional studies using different population are warranted to further validate ethnic differences on the impact of this polymorphism on PICH risk.

Only one study on women identified a positive association, therefore there was not enough power to investigate a potential gender interaction for the ACE I/D variant. Such a sex-specific influence may attribute to corticosteroid hormones, which play a crucial role in the renin-angiotensin-aldosterone system in a variety of tissues [41]. In future studies, more concentration on sex-dependent models should be taken into consideration to provide a more powerful analytical framework [42].

A potential publication bias may be present in the allele genetic model. Nevertheless, the combined risk estimate may not be changed just by modifying this bias using the trim and fill method. Though no significant publication bias for DD vs DI/II was found using the Begg's funnel plots or the Egger's test, the results should be explained with caution. Interpreting the $\mathrm{p}$ value in the setting of significant heterogeneity between studies may be problematic and limited.

There is a known positive association between hypertension and ICH [31]. Therefore, it is vital to know the HBP status of both $\mathrm{PICH}$ patients and controls. Questions like whether the patients were under ACE inhibitor usage or not should be known as well. We failed to analyze these issues due to lack of original data in this meta-analysis. Moreover, the combination of the polymorphisms and gene-environment interactions should be investigated. Despite difficulties in study design and the assessment of environmental factors, future case-control studies may help to resolve these questions if the studies include information on the status of hypertension, the use of ACE inhibitors and environmental exposures, such as smoking history. Patients who are at risk of mortality from PICH, as determined by ACE I/D polymorphism screening, may be chosen as good candidates for treatment with ACE inhibitors. However, an association between ACE I/D polymorphism and PICH mortality was not investigated as insufficient information (based on long term follow up for each patient) could not be universally provided by these studies.

Some limitations have already been considered when explaining the results in this meta-analysis. First, our study is under-powered to some degree due to its small sample size [43], and a genuine association between the ACE I/D polymorphism genotype and PICH susceptibility may fail to be detected due to the limited size. At the same time, it is difficult to reduce the rate of false discovery in all circumstances unless there is great similarity between study heterogeneity [44]. Therefore, more studies should be reviewed to obtain conclusive results. Second, the majority of the studies examined herein were from Asian populations, with only two of the studies examined from Caucasian populations, which may bias our results to be applicable only in Asian populations. Further population studies are required to investigate the association between the ACE I/D polymorphism and PICH risk in other races including African Americans and Latinos. Finally, the data were not subdivided into additional groups based on other variables, such as gender, due to the limitation of the original information for each patient. A more precise analysis could be performed if adjusted estimates were available in all studies.

\section{Conclusion}

In conclusion, the present study provides the most comprehensive meta-analysis to date on the assessment of the relationship between the ACE I/D polymorphism and PICH risk. The present study supports an association between ACE I/D polymorphic variant and PICH in Asians, but not in Caucasians. However, additional large-scale studies with well-matched controls and multi-ethnic populations are required to validate our conclusions.

\section{Supporting Information}

Figure S1 Forest plot of OR with 95\% CI for ACE I/D polymorphism in PICH susceptibility. (recessive model).

(TIF) 
Figure S2 Forest plot of OR with 95\% GI for ACE I/D polymorphism in PICH susceptibility. (allele model). (TIF)

Figure S3 Begg's funnel plot for publication bias in selection of studies of the ACE I/D polymorphism. (recessive model).

(TIF)

\section{References}

1. Qureshi AI, Tuhrim S, Broderick JP, Batjer HH, Hondo H, et al. (2001) Spontaneous intracerebral hemorrhage. N Engl J Med 344: 1450-1460.

2. Woo D, Broderick JP (2002) Spontaneous intracerebral hemorrhage: epidemiology and clinical presentation. Neurosurg Clin N Am 13: 265-279, v.

3. Broderick J, Connolly S, Feldmann E, Hanley D, Kase C, et al. (2007) Guidelines for the management of spontaneous intracerebral hemorrhage in adults: 2007 update: a guideline from the American Heart Association/ American Stroke Association Stroke Council, High Blood Pressure Research Council, and the Quality of Care and Outcomes in Research Interdisciplinary Working Group. Circulation 116: e391-413.

4. Flaherty ML, Woo D, Haverbusch M, Sekar P, Khoury J, et al. (2005) Racial variations in location and risk of intracerebral hemorrhage. Stroke 36: 934-937.

5. Inagawa $\mathrm{T}(2007)$ Risk factors for primary intracerebral hemorrhage in patients in Izumo City, Japan. Neurosurg Rev 30: 225-234; discussion 234.

6. Rigat B, Hubert C, Alhenc-Gelas F, Cambien F, Corvol P, et al. (1990) An insertion/deletion polymorphism in the angiotensin I-converting enzyme gene accounting for half the variance of serum enzyme levels. J Clin Invest 86: 1343 1346.

7. Dessi-Fulgheri P, Catalini R, Sarzani R, Sturbini S, Siragusa N, et al. (1995) Angiotensin converting enzyme gene polymorphism and carotid atherosclerosis in a low-risk population. J Hypertens 13: 1593-1596.

8. Fukuda D, Enomoto S, Nagai R, Sata M (2009) Inhibition of renin-angiotensin system attenuates periadventitial inflammation and reduces atherosclerotic lesion formation. Biomed Pharmacother 63: 754-761.

9. Eichel R, Khouri ST, Cohen JE, Ben-Hur T, Paniri R, et al. (2010) Prior use of angiotensin-converting enzyme inhibitors does not affect outcome in patients with intracerebral hemorrhage. Neurol Res 32: 905-909.

10. Peck G, Smeeth L, Whittaker J, Casas JP, Hingorani A, et al. (2008) The genetics of primary haemorrhagic stroke, subarachnoid haemorrhage and ruptured intracranial aneurysms in adults. PLoS One 3: e3691.

11. Zintzaras E (2010) The generalized odds ratio as a measure of genetic risk effect in the analysis and meta-analysis of association studies. Stat Appl Genet Mol Biol 9: Article21.

12. Robins J, Greenland S, Breslow NE (1986) A general estimator for the variance of the Mantel-Haenszel odds ratio. Am J Epidemiol 124: 719-723.

13. Mantel N, Haenszel W (1959) Statistical aspects of the analysis of data from retrospective studies of disease. J Natl Cancer Inst 22: 719-748.

14. DerSimonian R, Laird N (1986) Meta-analysis in clinical trials. Control Clin Trials 7: 177-188.

15. Zintzaras E, Ioannidis JP (2005) Heterogeneity testing in meta-analysis of genome searches. Genet Epidemiol 28: 123-137.

16. Ioannidis JP, Trikalinos TA, Zintzaras E (2006) Extreme between-study homogeneity in meta-analyses could offer useful insights. J Clin Epidemiol 59: 1023-1032.

17. Huedo-Medina TB, Sanchez-Meca J, Marin-Martinez F, Botella J (2006) Assessing heterogeneity in meta-analysis: $Q$ statistic or I2 index? Psychol Methods 11: 193-206.

18. Egger M, Davey Smith G, Schneider M, Minder C (1997) Bias in meta-analysis detected by a simple, graphical test. BMJ 315: 629-634

19. Stroup DF, Berlin JA, Morton SC, Olkin I, Williamson GD, et al. (2000) Metaanalysis of observational studies in epidemiology: a proposal for reporting. Metaanalysis Of Observational Studies in Epidemiology (MOOSE) group. JAMA 283: 2008-2012

20. Catto A, Carter AM, Barrett JH, Stickland M, Bamford J, et al. (1996) Angiotensin-converting enzyme insertion/deletion polymorphism and cerebrovascular disease. Stroke 27: 435-440.

21. Nakata Y, Katsuya T, Rakugi H, Takami S, Sato N, et al. (1997) Polymorphism of angiotensin converting enzyme, angiotensinogen, and apolipoprotein $\mathrm{E}$ genes in a Japanese population with cerebrovascular disease. Am J Hypertens 10: 1391-1395.
Figure S4 Begg's funnel plot for publication bias in selection of studies of the ACE I/D polymorphism. (allele model).

(TIF)

\section{Author Contributions}

Conceived and designed the experiments: LB. Analyzed the data: YS YL. Contributed reagents/materials/analysis tools: GYY. Wrote the paper: YS YL LTW. Adjudicated the data extraction: QS ZZ.

22. Lin JJ, Yueh KC, Lin GY, Chang DC, Chang CY, et al. (2000) Lack of association between angiotensin I-converting enzyme gene deletion polymorphism and cerebrovascular disease in Taiwanese. J Formos Med Assoc 99: 895901.

23. Slowik A, Turaj W, Dziedzic T, Haefele A, Pera J, et al. (2004) DD genotype of ACE gene is a risk factor for intracerebral hemorrhage. Neurology 63: 359-361.

24. Chen CM, Chen YC, Wu YR, Hu FJ, Lyu RK, et al. (2008) Angiotensinconverting enzyme polymorphisms and risk of spontaneous deep intracranial hemorrhage in Taiwan. Eur J Neurol 15: 1206-1211.

25. Li Y, Chen F, Zhou L, Coulter D, Chen C, et al. (2010) COC use, ACE/AGT gene polymorphisms, and risk of stroke. Pharmacogenet Genomics 20: 298-306.

26. Kalita J, Somarajan BI, Kumar B, Mittal B, Misra UK (2011) A study of ACE and ADD1 polymorphism in ischemic and hemorrhagic stroke. Clin Chim Acta 412: $642-646$.

27. Chowdhury AH, Kokubo Y, Yokoyama T, Yoshiike N, Mohammed QD, et al. (2000) Deletion polymorphism of ACE gene is associated with cortical stroke in the age group (less-than or equal to) 60 years in a Bangladeshi hospital-based study. CVD Prevention 3: 195-204

28. Rossi G, Rossi A, Sacchetto A, Pavan E, Pessina AC (1995) Hypertensive cerebrovascular disease and the renin-angiotensin system. Stroke 26: 17001706.

29. Griendling KK, Murphy TJ, Alexander RW (1993) Molecular biology of the renin-angiotensin system. Circulation 87: 1816-1828.

30. Egger M, Smith GD, Altman DG (2001) Meta-analysis in Context. London, England: BMJ Books.

31. Ariesen MJ, Claus SP, Rinkel GJ, Algra A (2003) Risk factors for intracerebral hemorrhage in the general population: a systematic review. Stroke 34: 20602065.

32. Appelros P, Stegmayr B, Terent A (2009) Sex differences in stroke epidemiology: a systematic review. Stroke 40: 1082-1090.

33. Balk EM, Bonis PA, Moskowitz H, Schmid CH, Ioannidis JP, et al. (2002) Correlation of quality measures with estimates of treatment effect in metaanalyses of randomized controlled trials. JAMA 287: 2973-2982.

34. Thompson SG (1994) Why sources of heterogeneity in meta-analysis should be investigated. BMJ 309: 1351-1355.

35. Egger M, Juni P, Bartlett C, Holenstein F, Sterne J (2003) How important are comprehensive literature searches and the assessment of trial quality in systematic reviews? Empirical study. Health Technol Assess 7: 1-76.

36. Tiret L, Rigat B, Visvikis S, Breda C, Corvol P, et al. (1992) Evidence, from combined segregation and linkage analysis, that a variant of the angiotensin Iconverting enzyme (ACE) gene controls plasma ACE levels. Am J Hum Genet 51: $197-205$.

37. Danser AH, Schalekamp MA, Bax WA, van den Brink AM, Saxena PR, et al (1995) Angiotensin-converting enzyme in the human heart. Effect of the deletion/insertion polymorphism. Circulation 92: 1387-1388.

38. Daemen MJ, Lombardi DM, Bosman FT, Schwartz SM (1991) Angiotensin II induces smooth muscle cell proliferation in the normal and injured rat arterial wall. Circ Res 68: 450-456.

39. Fleming I (2006) Signaling by the angiotensin-converting enzyme. Circ Res 98: 887-896.

40. Juo SH (2009) Genetics of carotid atherosclerosis. Front Biosci 14: 4525-4534.

41. Fischer M, Baessler A, Schunkert H (2002) Renin angiotensin system and gender differences in the cardiovascular system. Cardiovasc Res 53: 672-677.

42. Weiss LA, Pan L, Abney M, Ober C (2006) The sex-specific genetic architecture of quantitative traits in humans. Nat Genet 38: 218-222.

43. Sawcer S (2010) Bayes factors in complex genetics. Eur J Hum Genet 18: 746750 .

44. Hedges LV, Pigott TD (2001) The power of statistical tests in meta-analysis. Psychol Methods 6: 203-217. 